\title{
Radiative heat transfer in inhomogeneous, nongray, and anisotropically scattering media
}

\author{
Zhixiong Guo ${ }^{1}$, Shigenao Maruyama* \\ Institute of Fluid Science, Tohoku University, Katahira 2-1-1, Aoba-ku, Sendai 980-8577, Japan
}

Received 19 February 1999; received in revised form 17 September 1999

\begin{abstract}
Radiative heat transfer in three-dimensional inhomogeneous, nongray and anisotropically scattering participating media was investigated by using $\mathrm{REM}^{2}$ method. The accuracy of the method was verified by benchmark comparisons against the solutions of Monte Carlo and YIX methods in the case of three-dimensional inhomogeneous media and in the case of three-dimensional media composed of nongray $\mathrm{CO}_{2}$ gas and carbon particles with strong anisotropically scattering. The ray effect and the influences of radiation element division and spectral discretization were examined. The present predictions of heat flux, flux divergence and emissive power were found to be in good agreement with those by Monte Carlo and YIX methods. As an example of an application of engineering interest, radiative heat transfer in a boiler model with non-isothermal, nongray, and anisotropically scattering media is numerically studied. The distributions of radiative heat flux and flux divergences in the boiler furnace are obtained. It is found that larger heat fluxes appear at the wall near the flame and larger heat flux divergences exist around the boundary of the flame. The effect of particle radiation is small in the region downstream the flame. (C) 2000 Elsevier Science Ltd. All rights reserved.
\end{abstract}

\section{Introduction}

In recent decade, significant progress has been made in the development of solution methods for three-dimensional radiative heat transfer in absorbing, emitting and scattering participating media [1]. As pointed out by Farmer and Howell [2], however, it is usually a formidable task if the nongray and

\footnotetext{
* Corresponding author. Tel.: +81-22-217-5243; fax: + 8122-217-5243.

E-mail address: maruyama@ifs.tohoku.ac.jp (S. Maruyama).

${ }^{1}$ Present address: Department of Mechanical Engineering, Polytechnic University, 6 Metrotech Center, Brooklyn, NY 11201, USA.
}

anisotropically scattering characters of the medium are accounted for. The application to complex three-dimensional configurations is also difficult. The $\mathrm{REM}^{2}$ method proposed by Maruyama and Aihara [3] has been demonstrated in gray homogeneous media with complex configurations in Refs. [3,4]. Recently, it was developed to take the nongray and anisotropic scattering properties into account in three-dimensional homogeneous medium by Maruyama et al. [5]. However, it has only been verified in one-dimensional plane parallel plates with gray or nongray and anisotropic scattering media. As noted by Tong and Skocypec [1] in their recent summary on comparison of radiative transfer solutions, several numerical methods which predicted similar one-dimensional results failed to describe similar behavior in three-dimensional nongray and anisotropic scattering medium. Thus, the $\mathrm{REM}^{2}$ 


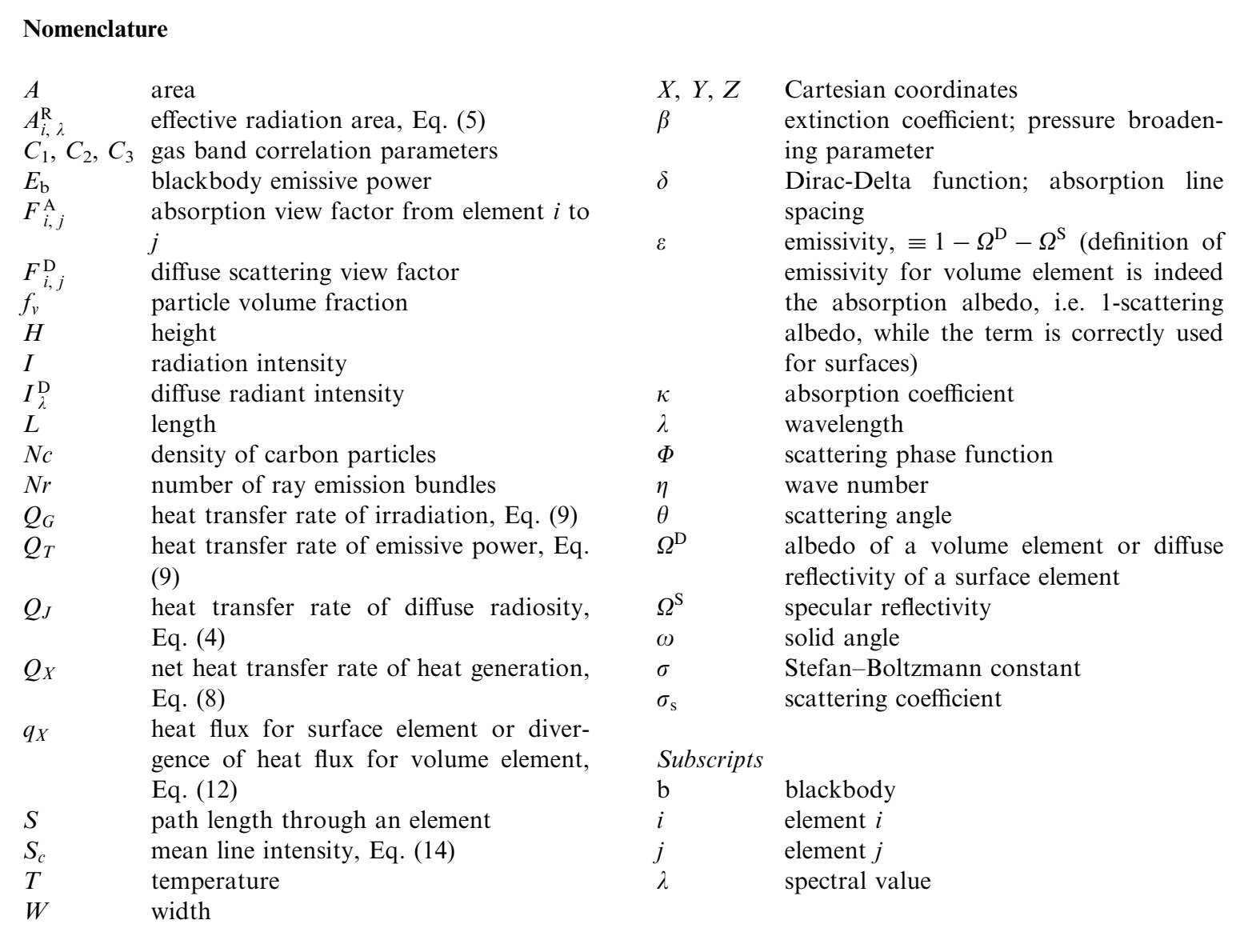

method has to be verified in three-dimensional nongray and anisotropically scattering media. Moreover, the method has not been applied to three-dimensional inhomogeneous medium which is usually encountered in industrial systems such as furnaces and combustion chambers. These motivate the present study.

To assess the current capability for solving nongray, anisotropically scattering three-dimensional radiation problems, a specific problem [1] was recently formulated for benchmark comparisons of solving methods. The problem considered threedimensional radiative heat transfer in a rectangular enclosure containing a mixture of anisotropically scattering carbon particles, nongray $\mathrm{CO}_{2}$ gas, and $\mathrm{N}_{2}$. Several solution techniques, such as Monte Carlo method [2], YIX method [6], and Generalized Zonal method [7], have been applied to solve this problem. The published results of these methods may be served as benchmark comparisons for other solutions.

Inhomogeneity is usually a basic feature in most engineering applications. In the $\mathrm{REM}^{2}$ method, the temperature and radiative properties are assumed to be uniform and constant in each radiation element. However, these properties can be inhomogeneous among different radiation elements. Hence, it can be easily applied to inhomogeneous participating media. Recently, Hsu and Farmer [8] presented benchmark solutions of radiative heat transfer within three-dimensional nonhomogeneous participating media by using the Monte Carlo and YIX methods. It is necessary for the present method to be verified in inhomogeneous participating medium. The numerical uncertainty of the present method, such as the effects of directional discretization and spatial discretization, should be examined.

Accurate prediction of thermal radiation in combustion chamber has been an important and active research subject in recent years [9-13]. It is of practical significance that the radiation in the flame and combustion systems are treated as nongray, anisotropically scattering and inhomogeneous due to the radiation of combustion gas and carbon particles and the non-uniform distributions of temperature and absorbing gaseous species. However, many investigators in 
combustion field do not take the nongray and anisotropically scattering characteristics into account. The assumption of isotropic scattering is somewhat questionable.

In the present treatise, the $\mathrm{REM}^{2}$ is developed to incorporate the spectral dependence of radiation properties, the anisotropically scattering of particles, and the inhomogeneous properties of participating media. The Elsasser narrow band model is employed in conjunction with the exponential wide band model for combustion gas radiation. The use of such a gas model is for the convenience of benchmark comparison. The present approach is first compared with the Monte Carlo and YIX methods in a three-dimensional inhomogeneous gray medium. Comparisons of the predictions of heat flux, flux divergence and emissive power are performed. The effects of ray emission bundles and element division mesh are examined. Then, the radiative heat transfer in three-dimensional rectangular media involving nongray $\mathrm{CO}_{2}$ gas and anisotropic scattering carbon particles is investigated. The accuracy of the present method is verified by benchmark comparisons with the published Monte Carlo and YIX calculations. The influence of spectral discretization is discussed. Finally, the radiative heat transfer is demonstrated in a boiler furnace with inhomogeneous and nongray radiation properties. The distributions of heat flux at the walls and heat flux divergence inside the boiler are obtained. The effect of particle volume fraction on radiative heat transfer in the furnace is discussed.

\section{Mathematical model}

Considering a radiation element of participating medium, the spectral radiation intensity $I_{\lambda}$ at $\vec{r}$ in the direction $\hat{s}$ can be formulated as

$$
\begin{aligned}
\frac{\mathrm{d} I_{\lambda}(\vec{r}, \hat{s})}{\mathrm{d} S}= & -\left(\kappa_{\lambda}+\sigma_{\mathrm{s}, \lambda}\right) I_{\lambda}(\vec{r}, \hat{s})+\kappa_{\lambda} I_{\mathrm{b}, \lambda}(T) \\
& +\frac{\sigma_{\mathrm{s}, \lambda}}{4 \pi} \int_{4 \pi} I_{\lambda}\left(\vec{r}, \hat{s}^{\prime}\right) \Phi_{\lambda}\left(\hat{s}^{\prime} \rightarrow \hat{s}\right) \mathrm{d} \omega,
\end{aligned}
$$

where $\kappa_{\lambda}$ and $\sigma_{\mathrm{s}, \lambda}$ are the spectral absorption and scattering coefficients, respectively, and $\Phi_{\lambda}\left(\hat{s}^{\prime} \rightarrow \hat{s}\right)$ is the phase function.

To simplify the problem, the following assumptions are introduced: (1) temperature, heat generation rate and refractive index in each element is constant and uniform; (2) scattering in the participating medium is isotropic, while scaling technique is employed for anisotropic scattering; (3) the scattering radiation distributes uniformly over the element. The third term of the right-hand side of Eq. (1) is then approximated as

$$
\begin{aligned}
& \frac{\sigma_{\mathrm{s}, \lambda}}{4 \pi} \int_{4 \pi} I_{\lambda}\left(\vec{r}, \hat{s}^{\prime}\right) \Phi_{\lambda}\left(\hat{s}^{\prime} \rightarrow \hat{s}\right) \mathrm{d} \omega \\
& \quad=\frac{\sigma_{\mathrm{s}, \lambda}}{4 \pi} \int_{4 \pi} I_{\lambda}\left(\vec{r}, \hat{s}^{\prime}\right) \mathrm{d} \omega=\sigma_{\mathrm{s}, \lambda} I_{\lambda}^{\mathrm{D}},
\end{aligned}
$$

where $I_{\lambda}^{\mathrm{D}}$ is the averaged diffuse radiant intensity. It is worth noting that $I_{\lambda}^{\mathrm{D}}$ is similar to diffuse radiosity that was used for radiation transfer of arbitrary diffuse and specular surfaces [14]. Introducing the extinction coefficient $\beta_{\lambda}$ and scattering albedo $\Omega$, Eq. (1) is rewritten as

$$
\begin{aligned}
\frac{\mathrm{d} I_{\lambda}(\vec{r}, \hat{s})}{\mathrm{d} S}= & \beta_{\lambda}\left[-I_{\lambda}(\vec{r}, \hat{s})+\left(1-\Omega_{\lambda}\right) I_{\mathrm{b}, \lambda}(T)\right. \\
& \left.+\Omega_{\lambda} I_{\lambda}^{\mathrm{D}}\right] .
\end{aligned}
$$

If the albedo $\Omega_{\lambda}$ is redefined as $\Omega_{\lambda}^{\mathrm{D}}$ and the specular reflectivity of surface element $\Omega_{\lambda}^{\mathrm{S}}$ is defined, a generalized form of the spectral radiant energy of element $i$, which may be either a volume or a surface element, can be expressed as follows:

$$
\begin{aligned}
& Q_{J, i, \lambda}(\hat{s})=\left[\left(1-\Omega_{i, \lambda}^{\mathrm{D}}-\Omega_{i, \lambda}^{\mathrm{S}}\right) I_{\mathrm{b}, i, \lambda}\left(T_{i}\right)\right. \\
& \left.\quad+\Omega_{i, \lambda} I_{i, \lambda}^{\mathrm{D}}\right] \int_{A_{i}(\hat{s})}\left[1-\exp \left(-\int_{0}^{S} \beta_{i, \lambda} \mathrm{d} S^{\prime}\right)\right] \mathrm{d} \omega \mathrm{d} A,
\end{aligned}
$$

where $\int_{0}^{S} \beta_{i, \lambda} \mathrm{d} S^{\prime} \gg 1$ stands for a surface element and $\Omega_{i}^{\mathrm{S}}=0$ for a volume element, respectively.

To simplify the integration in Eq. (4), we introduce an effective radiation area $A_{i, \lambda}^{\mathrm{R}}$ as

$$
\begin{gathered}
A_{i, \lambda}^{\mathrm{R}}=\frac{1}{\pi} \int_{A_{i}(\hat{s})}\left[1-\exp \left(-\int_{0}^{S} \beta_{i, \lambda} \mathrm{d} S^{\prime}\right)\right] \mathrm{d} \omega \mathrm{d} A \\
\approx \frac{1}{\pi} \int_{4 \pi} A_{i}(\hat{s})\left[1-\exp \left(-\int_{0}^{\bar{S}} \beta_{i, \lambda} \mathrm{d} S^{\prime}\right)\right] \mathrm{d} \omega,
\end{gathered}
$$

where an averaged thickness of the radiation element in the direction $\hat{s}$ was introduced as

$\bar{S} \equiv \frac{V}{A}(\hat{s})$,

in which, $V$ and $A(\hat{s})$ are the volume and area projected onto the surface normal to $\hat{s}$, respectively. It is worthy of noticing that $A_{i, \lambda}^{\mathrm{R}}$ is identical to the surface area for a surface element in which the reverse side is not incorporated, and $A_{i, \lambda}^{\mathrm{R}}$ is a function of wavelength for a nongray participating medium. The rate of spectral radiation energy emitted and isotropically scattered by the radiation element can be expressed in a generalized form as 
$Q_{J, i, \lambda} \equiv A_{i, \lambda}^{\mathrm{R}}\left(\varepsilon_{i} E_{\mathrm{b}, i, \lambda}+\Omega_{i, \lambda}^{\mathrm{D}} G_{i, \lambda}\right)$,

where $\varepsilon_{i}=1-\Omega_{i, \lambda}^{\mathrm{D}}-\Omega_{i, \lambda}^{\mathrm{S}}, E_{\mathrm{b}, i, \lambda}=\pi I_{\mathrm{b}, i, \lambda}, G_{i, \lambda}=\pi I_{i, \lambda}^{\mathrm{D}}$, and $Q_{J, i, \lambda}$ is the diffuse radiation transfer rate. The net rate of heat generation can be derived from the heat balance on the radiation element as

$Q_{X, i, \lambda}=A_{i, \lambda}^{\mathrm{R}} \varepsilon_{i}\left(E_{\mathrm{b}, i, \lambda}-G_{i, \lambda}\right)$.

The expressions of heat transfer rates of irradiation energy $Q_{G, i, \lambda}$ and emissive power $Q_{T, i, \lambda}$ of the radiation element are

$Q_{G, i, \lambda} \equiv A_{i, \lambda}^{\mathrm{R}} G_{i, \lambda}, \quad Q_{T, i, \lambda}=A_{i, \lambda}^{\mathrm{R}} \varepsilon_{i} E_{\mathrm{b}, i, \lambda}$.

If the system is consisted of $N$ volume and surface elements, then Eqs. (7) and (8) can be rewritten as

$\left.\begin{array}{c}Q_{J, i, \lambda}=Q_{T, i, \lambda}+\sum_{j=1}^{N} F_{j, i}^{\mathrm{D}} Q_{J, j, \lambda} \\ Q_{X, i, \lambda}=Q_{T, i, \lambda}-\sum_{j=1}^{N} F_{j, i}^{\mathrm{A}} Q_{J, j, \lambda}\end{array}\right\}$,

in which, the absorption view factor $F_{i, j}^{\mathrm{A}}$ and diffuse scattering view factor $F_{i, j}^{\mathrm{D}}$ are introduced [3]. It should be noted that the two view factors are functions of wavelength for a nongray participating medium. The heat transfer rate of spectral emissive power $Q_{T, i, \lambda}$ for each radiation element is given as a boundary condition. The unknown $Q_{X, i, \lambda}$ can be attained by solving Eq. (10). The total net rate of heat generation is obtained as

$Q_{X, i}=\int_{0}^{\infty} Q_{X, i, \lambda} \mathrm{d} \lambda$

The heat flux of a surface element or the heat flux divergence of a volume element is calculated by

$q_{X, i}=\frac{Q_{X, i}}{V_{i}}$

in which, $V_{i}=A_{i}$ for a surface element.

The radiation elements consisted of numerous polygons and polyhedrons can be produced by applying general purpose pre- and post-processor packages for the finite element method, e.g., the CAE software PATRAN. The radiation elements used in the present study are composed of arbitrary triangles, quadrilaterals, tetrahedrons, wedges, and hexahedrons. The view factors are calculated by using ray tracing method based on the ray emission model as described by Maruyama and Aihara [3].

\section{Spectral optical properties}

The Elsasser narrow band model is used in conjunction with the correlation parameters in Edwards wide band model [15] to determined the spectral absorption coefficients of $\mathrm{CO}_{2}$ and $\mathrm{H}_{2} \mathrm{O}$, which is given as follows,

$\kappa_{\eta}=\rho \frac{S_{c}}{\delta} \frac{\sinh (\pi \beta / 2)}{\cosh (\pi \beta / 2)-\cos \left[2 \pi\left(\eta-\eta_{c}\right) / \delta\right]}$,

where

$\frac{S_{c}}{\delta}=\frac{C_{1}}{C_{3}} \exp \left[-\left(\frac{a}{\omega}\right)\left|\eta-\eta_{c}\right|\right]$

$\beta=\frac{C_{2}^{2} P_{e}}{4 C_{1} C_{3}}$,

$\delta=30 C_{3} \quad(T=100 \mathrm{~K})$,

in which, $C_{1}, C_{2}, C_{3}$ and $P_{e}$ are the gas band correlation parameters [16]. The apparent extinction coefficient of element in direction $\hat{s}$ is evaluated based on the computation of gas transmittance through the element. Since each element is homogeneous, it is not necessary to employ Curtis-Godson method for the treatment of inhomogeneity. The spectral correlation is considered in the calculation of view factors.

The particles are assumed to be carbon spheres with a diameter of $30 \mu \mathrm{m}$ in all the models of the present study. Mie theory and the optical constants for carbon specimen number 2 in Foster and Howarth [17] were used to compute scattering and extinction efficiencies. The values are listed in Table 1 of Tong and Skocypec [1]. The scattering phase function was approximated by a delta-Eddington function [18] as

$\Phi(\Psi)=2 f \delta(1-\cos \theta)+(1-f)(1+3 g \cos \theta)$,

where $f=0.111$ and $g=0.215$.

Since Eq. (2) requires the assumption of isotropic scattering, the phase function of Eq. (17) is scaled to zeroth-order delta function approximation $[19,20]$. The scaled extinction coefficient $\beta_{P}^{*}$ and albedo $\Omega^{\mathrm{D}}$ are

$\beta_{P}^{*}=\beta_{P}\left(1-\frac{a_{1} \Omega}{3}\right), \quad \Omega^{\mathrm{D}}=\frac{\Omega\left(1-a_{1} / 3\right)}{1-a_{1} \Omega / 3}$,

in which $a_{1}=3(f+g-f \cdot g)$ when Eq. (17) is applied. Maruyama [19] and Guo and Maruyama [20] examined the accuracy of the zeroth-order approximation in one- and three-dimensional systems for forward and backward anisotropically scattering media.

The final extinction coefficient of the mixture of particles and gases is expressed as 
Table 1

Comparison of the present, YIX, and Monte Carlo methods in a three-dimensional inhomogeneous gray medium for case C1

\begin{tabular}{lllllll}
\hline & Present, $N r=45$ & Present, $N r=155$ & Present, $N r=561$ & Present, $N r=2141$ & YIX [8] & Monte Carlo [8] \\
\hline$X$ & & \multicolumn{7}{c}{ (a) Divergence of heat flux at $Y=Z=0$} \\
$-4 / 9$ & 0.07862 & 0.07867 & 0.07866 & 0.07866 & 0.07912 & 0.07974 \\
$-3 / 9$ & 0.15546 & 0.15557 & 0.15556 & 0.15556 & 0.15739 & 0.15866 \\
$-2 / 9$ & 0.23055 & 0.23076 & 0.23075 & 0.23074 & 0.23482 & 0.23673 \\
$-1 / 9$ & 0.30408 & 0.30450 & 0.30446 & 0.30445 & 0.31163 & 0.31433 \\
0 & 0.37664 & 0.37731 & 0.37733 & 0.37730 & 0.38842 & 0.39192 \\
& & & & & \\
-4 & & & (b) Surface heat flux at $X=-0.5$ and $Y=0$ & & 0.01214 \\
$-3 / 9$ & 0.01559 & 0.01196 & 0.01221 & 0.01210 & 0.01219 \\
$-2 / 9$ & 0.01808 & 0.01571 & 0.01569 & 0.01550 & 0.01589 & 0.01564 \\
$-1 / 9$ & 0.02065 & 0.01867 & 0.01860 & 0.01851 & 0.01877 & 0.01892 \\
0 & 0.02199 & 0.02087 & 0.02069 & 0.02062 & 0.02107 & 0.02103 \\
& 0.02186 & 0.02184 & 0.02155 & 0.02192 & 0.02202 \\
\hline
\end{tabular}

$\beta=\sum_{k=1}^{N_{\mathrm{g}}} \beta_{\mathrm{g} k}+\beta_{P}^{*}$,

where $\beta_{\mathrm{g} k}$ is the apparent extinction coefficient of the $k$ th gas, and $N_{\mathrm{g}}$ is the total number of gas components.

\section{Results and discussion}

\subsection{Cube with inhomogeneous gray media}

The radiative heat transfer in a unit cube with inhomogeneous gray media is investigated and compared with the results of other solutions. The geometry is shown in Fig. 1, in which $L=H=W=1$. All the walls are black. The optical thickness $(\tau=$ extinction coefficient $\times$ the side length, $W$ ) distribution is given by

$\tau=a\left(1-\frac{|X|}{0.5}\right)\left(1-\frac{|Y|}{0.5}\right)\left(1-\frac{|Z|}{0.5}\right)+b$.

A linear anisotropic scattering phase function is assumed:

$\Phi(\mu)=1+a_{1} P_{1}(\cos \mu)$,

where $P_{1}$ is the first-order term of Legendre series, and $a_{1}$ is the coefficient which can also be used in Eq. (18) when isotropic scaling is adopted. The scattering albedo is set as 0.9 . It should be noted that the deltaEddington phase function is employed in most of the present study except in this subsection. It is also worthy to point out that all the variables in this subsection do not have units. Two different cases are selected for study. In case C1: $a=0.9, b=0.1, a_{1}=0$, blackbody emissive power in the medium is given as unity, and all the walls are cold. In case C2: $a=5$, $b=5, a_{1}=1$, the radiative equilibrium is assumed, the wall at $X=-0.5$ has the black body emissive power and the rest walls are cold.

The predicted divergence of heat flux and surface heat flux for case $\mathrm{C} 1$ are compared with the calculations of Monte Carlo and YIX methods [8] in Table 1 . The cubic medium was divided into $9 \times 9 \times 9$ volume elements in the present calculations. The effect of ray emission bundles is also shown in Table 1. For all ray emission numbers ranged from $N r=45$ to $N r=2141$, the general differences of heat flux and flux divergence between the present method and the Monte Carlo and YIX methods are within 2\%.

The influence of volume element division is displayed in Fig. 2(a) and (b) for case C2. Three sets of

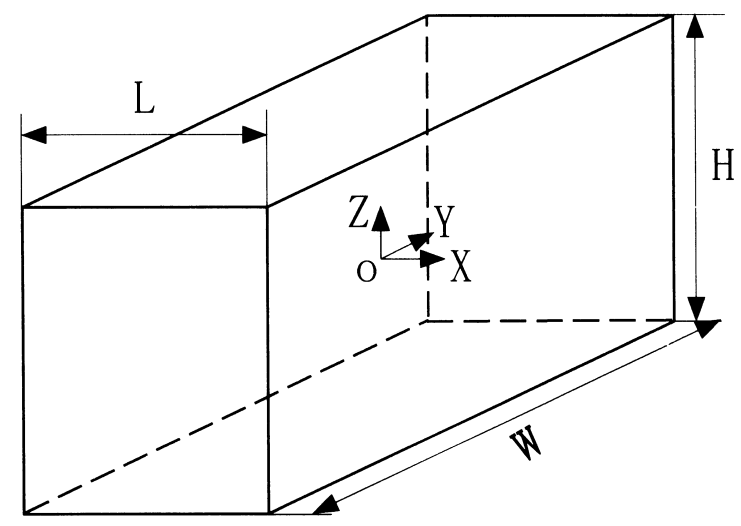

Fig. 1. Geometry of a three-dimensional rectangular medium. 
volume element mesh are selected for comparison and the ray emission number is set as 2141 . The results are also compared with the available data of Monte Carlo method [8]. In Fig. 2(a), the distributions of emissive power along $X$-direction at $Y=Z=0$ are shown. It is seen that the influence of volume element mesh on the emissive power is small. The largest difference of emissive power between coarse mesh $(5 \times 5 \times 5)$ and fine mesh $(19 \times 19 \times 19)$ is approximately $4 \%$. Even in the case of coarse mesh $(5 \times 5 \times 5)$, the present predictions are in good agreement with those by the Monte Carlo method. In the case of fine mesh $(19 \times 19 \times 19)$, the general difference of emissive power between the present method and the Monte Carlo method is $1 \%$. The influence of volume element division on radiative heat flux is illustrated in Fig. 2(b). It is found that the influence of volume element mesh is larger on the heat flux in the walls than on the emissive power inside the medium. The difference of heat flux between the coarse mesh $(5 \times 5 \times 5)$ and fine mesh $(19 \times 19 \times 19)$ ranges from $5 \%$ to $12 \%$ depending on the locations. The largest difference of heat flux between the present method and the Monte Carlo method is less than 2\%

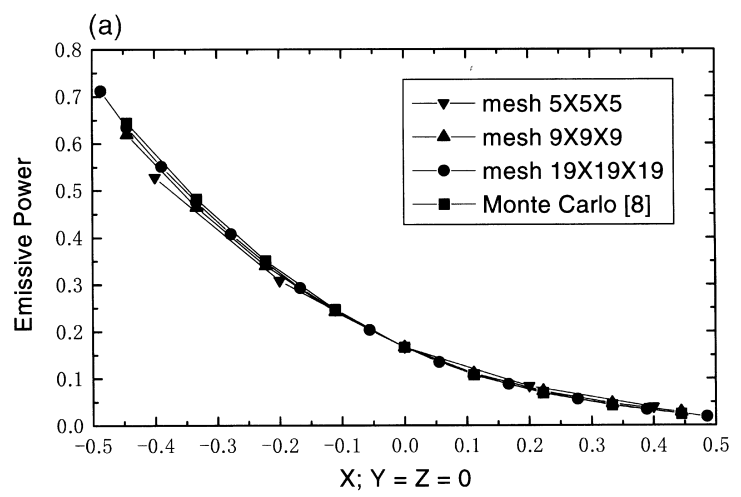

(b)

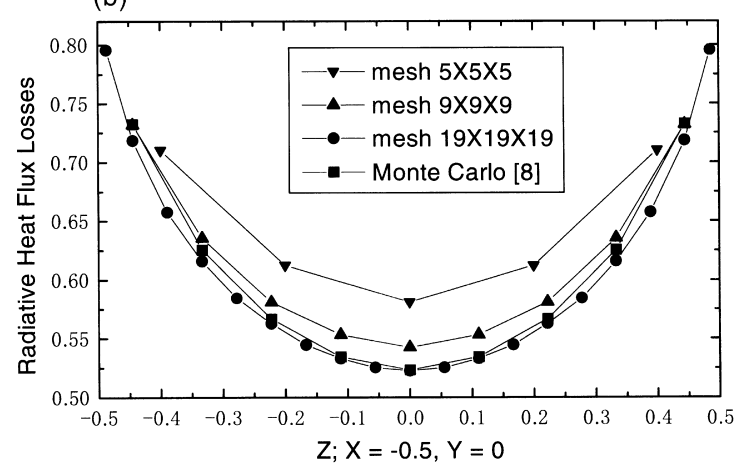

Fig. 2. Influence of element division on radiative heat transfer in case $\mathrm{C} 2$; (a) emissive power along $X$-direction at $Y=Z=0$, (b) radiative heat flux along $Z$-direction at wall $X=-0.5$ and $Y=0$. for fine mesh $(19 \times 19 \times 19)$ and less than $5 \%$ for intermediate mesh $(9 \times 9 \times 9)$. The finer is the element mesh grid, the more accurate is the prediction. However, the present method gives reasonably accurate solution even with coarse radiation element mesh.

As pointed out by Chai et al. [21], the ray effect at the cold walls is serious in radiative transfer analysis of a complex geometry when one wall is hot and the other walls are cold. The influence of ray emission number is demonstrated in Fig. 3 for case $\mathrm{C} 2$, in which one wall is hot and the scattering is anisotropic. The profiles of radiative heat flux along the cold wall $X=0.5$ and $Y=0$ are illustrated for $N r=45,155$, 561 and 2141, respectively. The element mesh grid is $9 \times 9 \times 9$. It is seen that the general difference between the predictions of a large number of ray emissions $(N r=2141)$ and a small number of ray emissions $(\mathrm{Nr}=45)$ is approximately $5 \%$, while the largest difference of $15 \%$ is found at the corners of the cube. When the number of ray emissions is not less than $N r=155$, the ray effect is small. However, the ray effect is more obvious in case $\mathrm{C} 2$ than in case $\mathrm{C} 1$, in which the scattering is isotropic and all the walls are cold. Nevertheless, the ray effect, which causes errors by the discrete ordinate method [21], is minimized in the present method. This is because the calculation of view factors is corrected using a method similar to that of Omori et al. [22] and the number of ray emission in the present solution is usually much larger than that used in the discrete ordinate method.

\subsection{Rectangular enclosure with nongray media}

The accuracy of the present method is then verified for three-dimensional nongray and anisotropic scattering media. The geometry and coordinates also shown in Fig. 1, but with $L=2 \mathrm{~m}, H=3 \mathrm{~m}$ and $W=5 \mathrm{~m}$. The medium is a mixture of $\mathrm{CO}_{2}$ and $\mathrm{N}_{2}$ gases and carbon particles. The pressure of the total mixture is

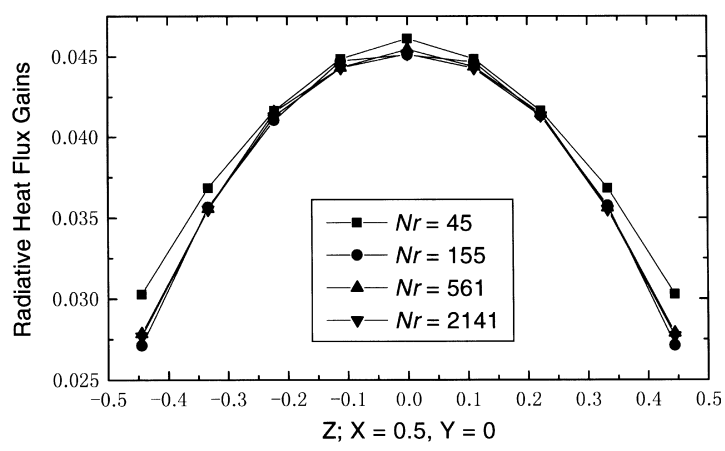

Fig. 3. Ray effect on radiative heat flux along $Z$-direction at cold wall $X=0.5$ and $Y=0$ in case C2. 
specified at 1 atm, with a volume fraction of $\mathrm{CO}_{2}$ of 0.21 . The temperature of the medium is constant and uniform at $1000 \mathrm{~K}$. The enclosure is covered with black and cold surfaces. A one-eighths symmetric analysis model, in which three symmetric surfaces are specified as totally specular reflection of $\Omega^{\mathrm{S}}=1$, is considered in the present calculations. The analysis model includes 216 volume elements, 108 black surface elements and 108 perfect mirror elements. The number of ray emissions from each element is 155 .

The benchmark comparisons against the Monte Carlo and YIX solutions are shown in Fig. 4 for the predictions of radiative heat flux for three levels of carbon particle density. It is seen that the present calculations are in good agreement with the simulations of Monte Carlo method [2] and YIX method [6] for all three levels of carbon density. The present results are very close to the calculations of Monte Carlo method for optical thin $\left(N c=2.0 \times 10^{7}\right.$ particles $\left./ \mathrm{m}^{3}\right)$ and optical thick $\left(N c=2.0 \times 10^{9}\right.$ particles $\left./ \mathrm{m}^{3}\right)$ participating media. The difference of heat flux between these two solutions is generally $5 \%$. For the medium with intermediate optical thickness $\left(N c=2.0 \times 10^{8}\right.$ particles $\left./ \mathrm{m}^{3}\right)$, the difference of heat flux between the present method and Monte Carlo method becomes larger in the vicinity of boundaries. The largest difference of the predicted heat flux is approximately $18 \%$.

The benchmark comparison is extended to Fig. 5, in which the divergence of radiative flux is compared between the present simulation and the Monte Carlo computation [2]. It is seen that the difference of flux

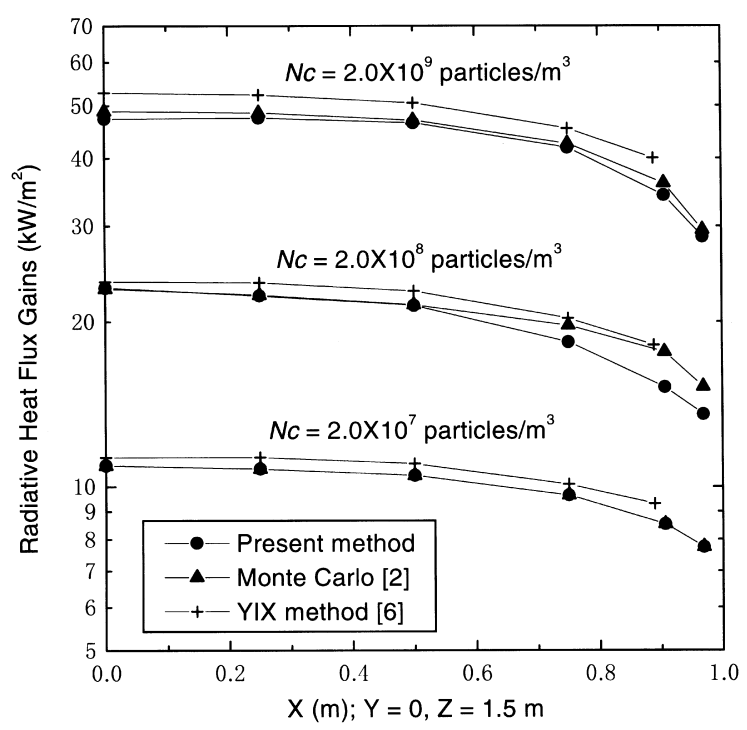

Fig. 4. Benchmark comparisons of radiative heat flux in three-dimensional enclosure with mixture of $\mathrm{CO}_{2}$ gas and carbon particles for three levels of particle density. divergence between the two solutions is basically within $10 \%$. However, $20 \%$ difference exists in the vicinity of boundaries. The difference of the radiative transfer predictions between any two solutions of the present method, Monte Carlo method and YIX method is much larger in nongray medium than in gray medium. This may be attributed to the different spectral integration techniques used in the three different methods as noticed by Hsu and Farmer [8].

The computation efficiency is also of major concern in radiation transfer calculations of three-dimensional configurations. In the present computations, a personal computer VT-Alpha/533 was employed, and the number of spectral discretization was set to be 100 . The total CPU time including the calculations of view factors and radiative transfer is approximately $240 \mathrm{~s}$. When the particle density increases, i.e., the participating media changes from optical thin $\left(N c=2.0 \times 10^{7}\right.$ particles $\left./ \mathrm{m}^{3}\right)$ to optical thick $\left(N c=2.0 \times 10^{9}\right.$ particles/ $\mathrm{m}^{3}$ ), the CPU time is slightly decreased. This is due to the ray tracing time decreases as the optical length of the medium increases. This tendency is different from the YIX method, in which the CPU time increases as the optical thickness of the medium increases.

The influence of spectral discretization is shown in Fig. 6 for the same gas and particle mixture with lower level of particle density $\left(N c=2.0 \times 10^{7}\right.$ particles $\left./ \mathrm{m}^{3}\right)$. The total spectrum ranges from 100 to $10000 \mathrm{~cm}^{-1}$. The spectrum was divided into 50, 100, 400, and 1000 sub-bands, respectively. It is seen from Fig. 6(a) that, the case of 100 sub-bands overestimates the radiative heat flux by $3-5 \%$ compared with the case of fine spectral discretization (1000 sub-bands). The overestimation increases $6-10 \%$ when the spectral discretization

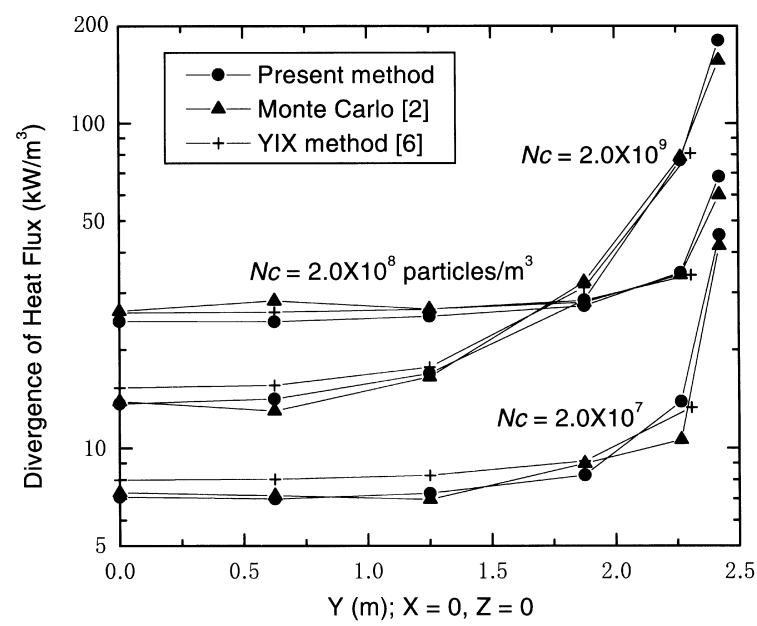

Fig. 5. Benchmark comparisons of heat flux divergence in three-dimensional enclosure with mixture of $\mathrm{CO}_{2}$ gas and carbon particles for three levels of particle density. 
further decreases to 50 sub-bands. However, the difference of heat flux between 400 sub-bands and 1000 subbands is minor. From Fig. 6(b), it is seen that the influence of spectral discretization on the divergence of heat flux is not as large as on the surface heat flux. The influence becomes strong at the locations near the boundary, where $4 \%$ difference of flux divergence is detected between 100 and 1000 sub-bands. We have also studied the influence of spectral discretization for larger particle density. We found that the influence becomes weak when the particle density increases. This is because the spectral-dependence of particle radiation is weak.

The CPU time for the calculation of nongray radiative heat transfer is linearly proportional to the number of spectral discretization. It is, therefore, valuable to know the trade-off between accuracy and efficiency for the calculation of nongray radiative heat transfer in complex configurations. When the particle radiation is comparable to or stronger than the gas radiation in the media containing mixture of gases and particles such as in coal-flamed or oil-flamed furnaces, reason-

(a)
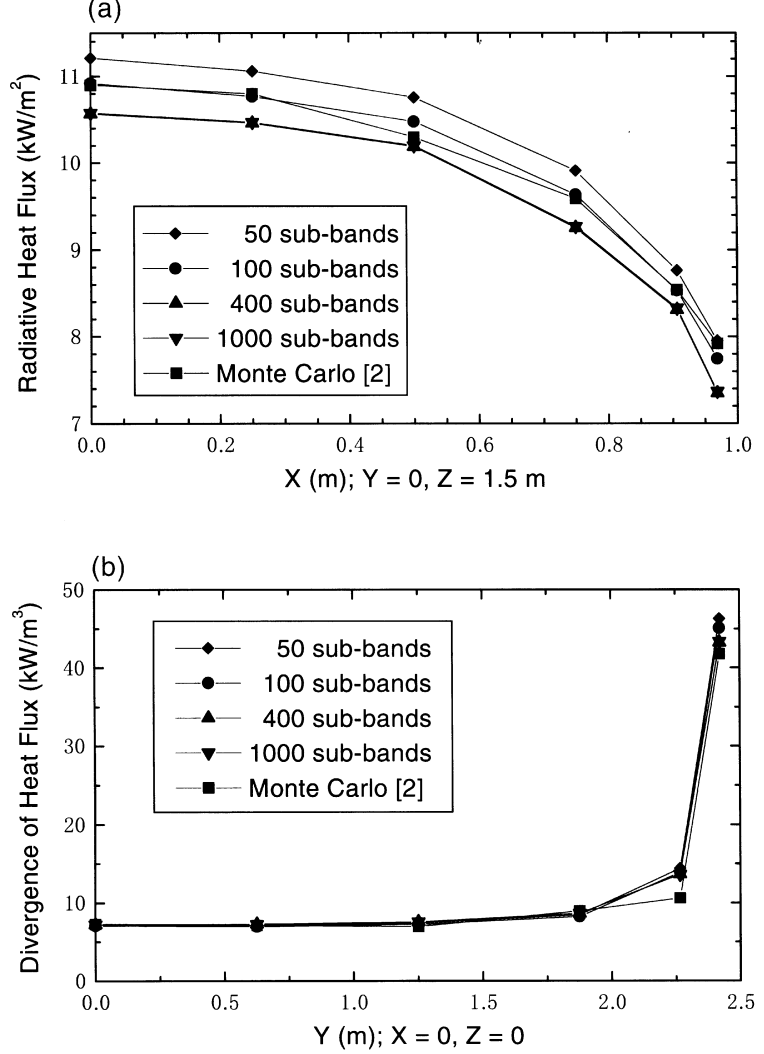

Fig. 6. Influence of spectral discretization on radiative heat transfer in three-dimensional enclosure with mixture of $\mathrm{CO}_{2}$ gas and carbon particles for $N c=2.0 \times 10^{7}$ particles $/ \mathrm{m}^{3}$. able accuracy and high computation efficiency can be achieved with a relative coarse spectral discretization.

The present REM $^{2}$ method is verified by benchmark comparisons against the Monte Carlo and YIX methods. However, it should be reminded that even Monte Carlo method is subject to statistical error. No numerical method is currently well acknowledged to have priority over other methods in the field of radiative transfer in three-dimensional inhomogeneous, nongray, and anisotropic scattering media.

\subsection{Radiation transfer in a boiler model}

As an example of application of engineering interest, radiative heat transfer in a boiler model as shown in Fig. 7 is investigated. The boiler is operated at atmospheric pressure. The combustion gas is composed of $\mathrm{CO}_{2}, \mathrm{H}_{2} \mathrm{O}$ and $\mathrm{N}_{2}$, and the mole fractions of $\mathrm{CO}_{2}$ and $\mathrm{H}_{2} \mathrm{O}$ are assumed to be 0.119 and 0.085 , respectively. The composition of the combustion gas is assumed to be uniform all over the boiler. The temperature of the boiler wall is $623 \mathrm{~K}$, except $813 \mathrm{~K}$ is assumed in the gas exit wall. All walls are diffuse with emissivity of 0.8. Following the study of Aoki et al. [23], a non-uniform temperature profile inside the boiler is assumed as follows.

when $Z \geq H_{1}$
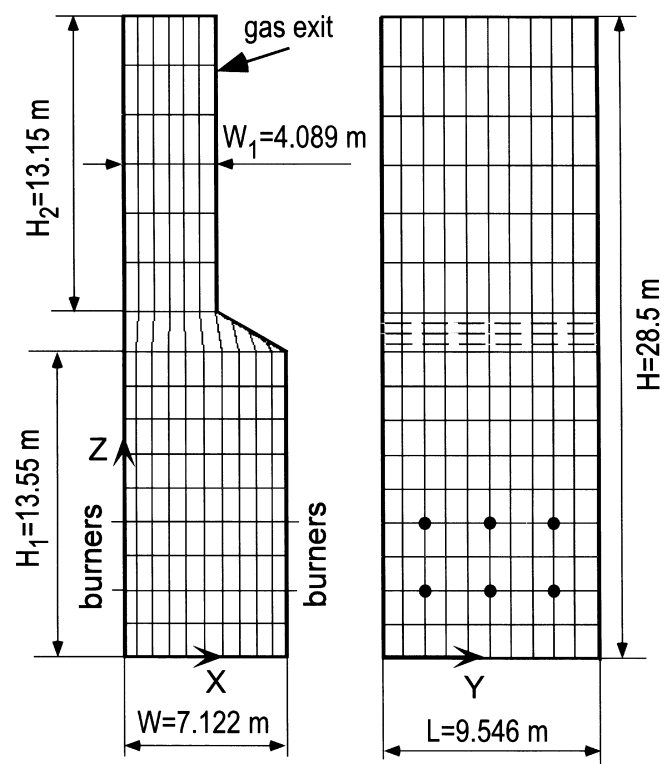

Fig. 7. Dimensions and element mesh grid arrangement of a boiler furnace. 


$$
\begin{aligned}
T= & T_{0}\left[a_{1}\left(1-\frac{\left|2 X-W_{1}\right|}{2 W-W_{1}}\right)\right. \\
& \left.\times\left(1-\frac{|2 Y-L|}{2 L}\right)\left(1-\frac{\left|Z-H_{1}\right|}{H-H_{1}}\right)+b_{1}\right]
\end{aligned}
$$

when $Z<H_{1}$

$$
\begin{aligned}
T= & T_{0}\left[a_{2}\left(1-\frac{|2 X-W|^{3}}{W^{3}}\right)\right. \\
& \left.\times\left(1-\frac{|2 Y-L|}{10 L}\right)\left(1-\frac{\left|2 Z-H_{1}\right|}{10 H_{1}}\right)+b_{2}\right]
\end{aligned}
$$

in which, $a_{1}=1, b_{1}=2.5, a_{2}=2.8, b_{2}=1$, and the input fuel temperature $T_{0}=563 \mathrm{~K}$. The soot inception region in flame is found to occur at the high temperature edge with maximum intensity in a region at 1300 $1650 \mathrm{~K}$ according to the measurement of Smyth et al. [24]. The carbon particles in the present model are assumed to be produced uniformly in a region where $1000 \mathrm{~K} \leq T \leq 2000 \mathrm{~K}$ and $H_{1} / 9 \leq Z \leq 6 H_{1} / 9$. Three cases of particle volume fraction of $f_{v}=10^{-6}, 10^{-5}$ and $10^{-4}$ are studied.

The model is specified to simulate the example of a $125 \mathrm{MW}_{\mathrm{e}}$ boiler furnace using heavy oil as the fuel as studied by Aoki et al. [23]. It should be mentioned that the distributions of gases and particles, and the temperature profiles in this model are not realistic. We have adopted this model to assess the applicability of the present method in analyzing radiative transfer in complex configurations with inhomogeneous, nongray and anisotropically scattering participating media.

The arrangement of radiation element is also shown in Fig. 7. In actual calculation, a half analysis model is employed since the boiler is symmetric along the center plane of $Y=L / 2$. The number of spectral discretization is set as 100 and the number of ray emissions from each element is selected as 155 based on the previous analysis of sensitivity.

The distributions of normalized heat flux $\left(q_{x} / \sigma T_{n}^{4}\right.$, $\left.T_{n}=2000 \mathrm{~K}\right)$ at the wall are illustrated in Fig. 8 for three levels of particle volume fraction. It is seen that larger heat fluxes are distributed at the wall near the flame region, while the heat fluxes on the surfaces in pure combustion gas and gas exit regions are very low. This is particularly obvious when the particle volume fraction is larger $\left(f_{v}=10^{-4}\right)$. It reveals that the radiation from high temperature particles may be much stronger than that from high temperature combustion gas in highly particle-laid flames. The heat flux at the wall near the flame is strongly influenced by the particle volume fraction. The larger the particle volume fraction is, the higher the magnitude of heat flux. The largest values of normalized heat flux are $0.198,0.321$ and 0.617 , respectively for $f_{v}=10^{-6}, 10^{-5}$ and $10^{-4}$. However, the heat flux is slightly influenced by the particle volume fraction at the top wall of the furnace, which is far away from the flame and the radiation there is mainly from the combustion gas. Thus, it is seen that the distributions of radiative heat flux at the walls of the upper part of the furnace are similar for all three levels of particle volume fraction. When the particle volume fraction increases from $f_{v}=10^{-6}$ to $f_{v}=10^{-4}$, the value of heat flux is increased by only $5-15 \%$ at those regions.

The distributions of normalized heat flux divergence $\left(q_{x} W / \sigma T_{n}^{4}\right)$ at the central plane of $Y=L / 2$ inside the boiler are depicted in Fig. 9 for three levels of particle volume fraction. It is also seen that the influence of particle volume fraction is very weak in the region of pure combustion gas downstream of the flame, and the distributions of flux divergence are similar in that region for all three levels of particle volume fraction. However, the divergence of heat flux at the locations around the flame boundaries increases obviously as the particle volume fraction increases. Larger values of flux divergences are found at the corners of the flame for the cases of $f_{v}=10^{-5}$ and $10^{-4}$. From the center of the flame to the boundary of the boiler, the flux divergence becomes larger until a location around the boundary of the flame. Then, the divergence of heat flux decreases from the boundary of the flame to the wall. Negative values of divergence of heat flux appear in the fuel- and air-input regions. This means that the radiative heat from the flame is absorbed in those areas because of the absorption of low temperature combustion gas, which is cooled by the input of very low temperature fuel and air $(563 \mathrm{~K})$. Therefore, the heat fluxes are much lower at the wall $(X=W)$ near the fuel- and air-input region than at the wall $(Y=0)$ near the flame as shown in Fig. 8.

\section{Conclusions}

The REM $^{2}$ method has been developed to investigate radiative heat transfer in inhomogeneous, nongray, and anisotropically scattering media with complex geometry for the first time. The accuracy and sensitivity of the method was evaluated by benchmark comparisons against the published calculation results of Monte Carlo and YIX methods in three-dimensional radiative transfer problems. The general difference of the predicted radiative heat transfer between the present method and the Monte Carlo and YIX methods was $2 \%$ for gray inhomogeneous media, while a general difference of $10 \%$ was found for participating media containing nongray gas and anisotro- 

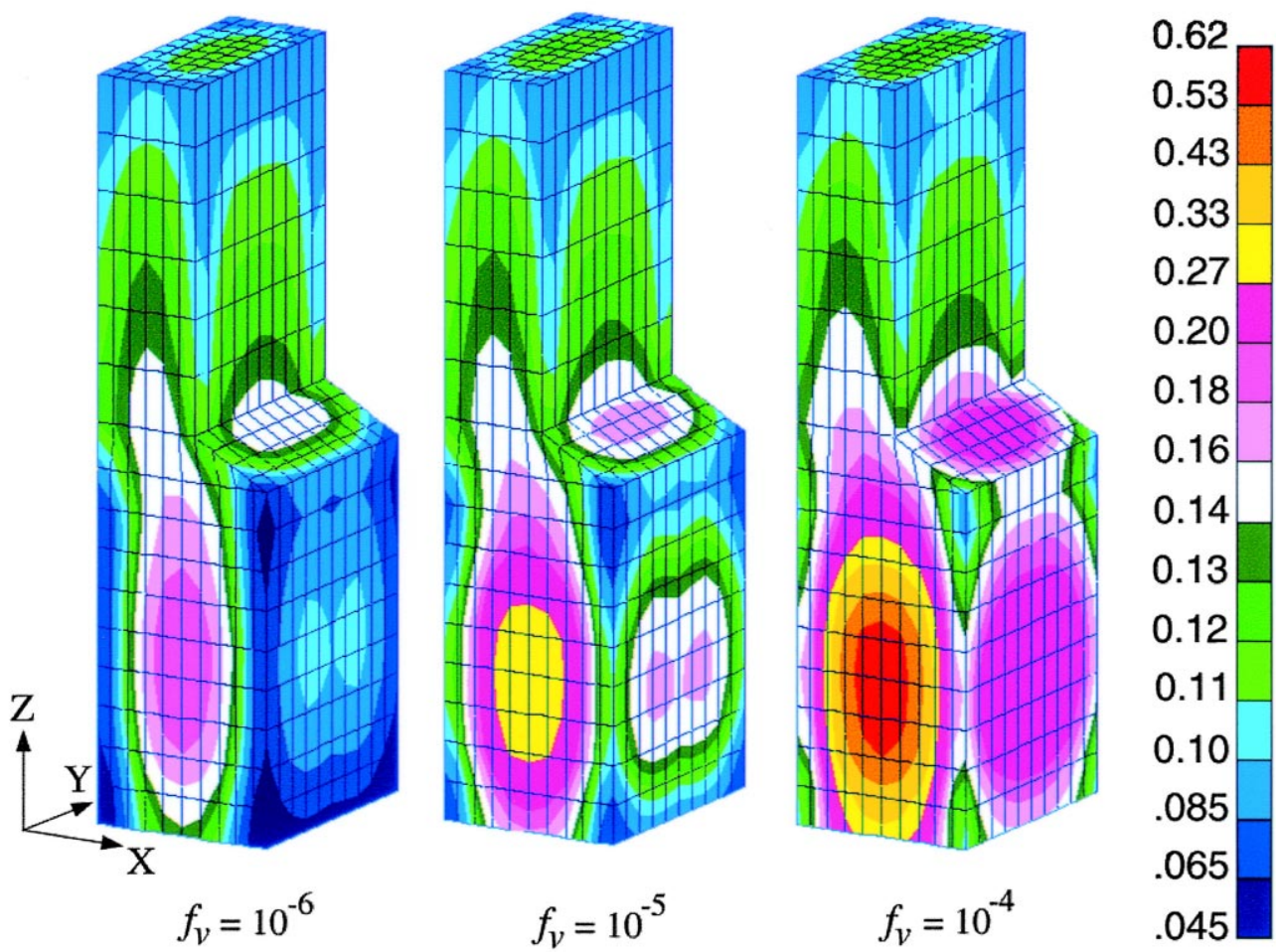

Fig. 8. Distributions of normalized radiative heat flux at the wall of the boiler furnace for three levels of particle volume fraction.

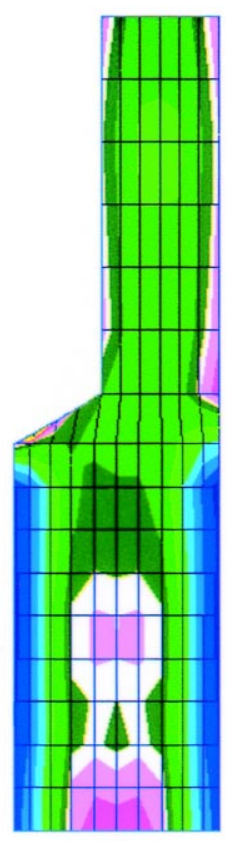

$$
f_{v}=10^{-6}
$$

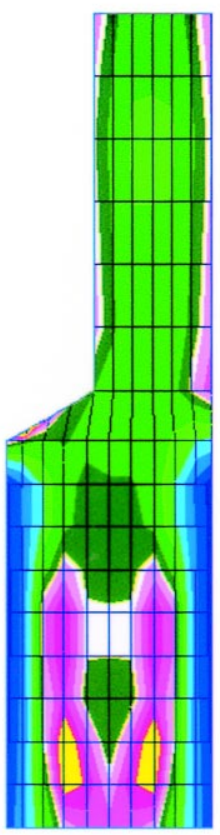

$f_{v}=10^{-5}$

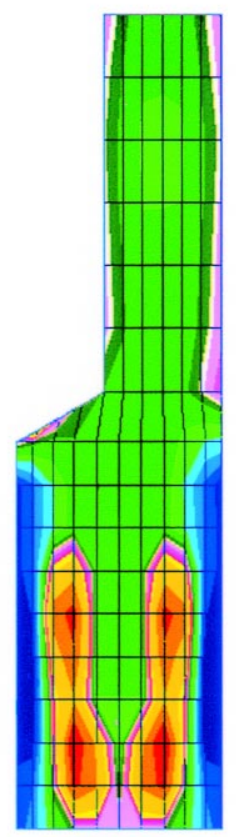

$f_{v}=10^{-4}$
2.62 2.20

1.75

1.25

1.10

1.00

0.90

0.80

0.75

0.65

0.45

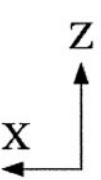

0.25

0.0

$-.25$

-.50
-.75

Fig. 9. Distributions of normalized divergence of heat flux at central plane of $Y=L / 2$ in the boiler furnace for three levels of particle volume fraction. 
pically scattering particles. The present study clarified the accuracy of REM ${ }^{2}$ method.

The influences of ray emission number, radiation element number and spectral discretization were examined. It is found that the ray effect is obvious at the boundaries of the walls for a three-dimensional anisotropically scattering medium. However, the ray effect can be minimized by the increase of the number of ray emission bundles. The increase of ray emission number just increases the computational time of view factors, but does not influence that of radiative transfer. Three sets of element division mesh grid for a cubic medium were selected to study the influence of radiation element number. It is found that a coarse mesh grid may overestimate the radiative heat flux. However, the tendency of the profiles of heat flux and emissive power for a coarse mesh grid is in reasonably good agreement with that of a fine mesh grid. The influence of spectral discretization is important for gas radiation. For a medium containing mixture of gases and particles, a relative coarse spectral discretization gives reasonable accurate predictions when the radiation of particles is not weaker than the radiation of gases. It is important to compromise the trade-off between the accuracy and the computation efficiency which are strongly dependent on the numbers of ray emission, element division and spectral discretization.

The radiative heat transfer in a boiler model with nongray combustion gas, anisotropic scattering particles and non-uniform temperature profiles was investigated. The complex configuration of the boiler was easily dealt with unstructured radiation elements composed of numerous polygons and polyhedrons. The distributions of heat flux and flux divergence were obtained. It is found that the heat fluxes are larger at the walls near the flame, where a number of radiation particles exist. The magnitude of heat flux divergence at the boundaries of the flame is larger than other regions inside the boiler. As the particle volume fraction increases, the magnitudes of both radiative heat flux and flux divergence increase. However, the influence of particle radiation is small in the pure combustion gas region far downstream of the flame. A part of radiative heat from the flame is absorbed by the low temperature combustion gas in the fuel- and air-input region.

\section{References}

[1] T.W. Tong, R.D. Skocypec, Summary on comparison of radiative heat transfer solutions for a specified problem, Developments in Radiative Heat Transfer, ASME HTD 203 (1992) 253-258.

[2] J.T. Farmer, J.R. Howell, Monte Carlo prediction of radiative heat transfer in inhomogeneous, anisotropic, nongray media, J. Thermophysics Heat Transfer 8 (1994) 133-139.

[3] S. Maruyama, T. Aihara, Radiation heat transfer between arbitrary three-dimensional absorbing, emitting and scattering media and specular and diffuse surfaces, ASME J. Heat Transfer 119 (1997) 129-136.

[4] S. Maruyama, M. Higano, Radiative heat transfer of torus plasma in large helical device by generalized numerical method $\mathrm{REM}^{2}$, Energy Conversion and Management 38 (1997) 1187-1195.

[5] S. Maruyama, Z. Guo, Radiation heat transfer in arbitrary configuration with nongray absorbing, emitting and anisotropic scattering media, ASME J. Heat Transfer 121 (1999) 722-726.

[6] P.F. Hsu, Z. Tan, J.R. Howell, Application of the YIX method to radiative heat transfer within a mixture of highly anisotropic scattering particles and non-gray gas, Developments in Radiative Heat Transfer, ASME HTD 203 (1992) 285-300.

[7] W.W. Yuen, A.K. Ma, E.E. Takara, Evaluation of radiative heat transfer using the generalized zonal method and the absorption mean beam length concept, Developments in Radiative Heat Transfer, ASME HTD 203 (1992) 265-273.

[8] P.F. Hsu, J.T. Farmer, Benchmark solution of radiative heat transfer within nonhomogeneous participating media using the Monte Carlo and YIX method, J. Heat Transfer 119 (1997) 185-188.

[9] W.A. Fiveland, R.A. Wessel, Numerical model for predicting performance of three-dimensional pulverized-fuel flamed furnaces, J. Eng. Gas Turbines Power 110 (1988) $117-126$

[10] B.W. Butler, M.K. Dension, B.W. Webb, Radiation heat transfer in a laboratory-scale, pulverized coal-fired reactor, Experimental Thermal Fluid Science 9 (1994) 69-79.

[11] H. Hayasaka, N. Matsumura, K. Kudo, Improvement of thermal efficiency of a furnace for thermal electric power plant (effect of division walls), Trans. Jpn. Soc. Mech. Engr., Ser. B 62 (1996) 2807-2811.

[12] K. Kudo, A. Kuroda, T. Saito, M. Oguma, Application of a method for solving inverse radiative load problems to design of furnaces, Trans. Jpn. Soc. Mech. Engr., Ser. B 62 (1996) 3131-3136.

[13] N. Selcuk, N. Kayakol, Evaluation of discrete ordinates method for radiative transfer in a rectangular furnaces, Int. J. Heat Mass Transfer 40 (1997) 213-222.

[14] S. Maruyama, Radiation heat transfer between arbitrary three-dimensional bodies with specular and diffuse surfaces, Numerical Heat Transfer, Part A 24 (1993) 181196.

[15] D.K. Edwards, A. Balakrishnan, Thermal radiation by combustion gases, Int. J. Heat Mass Transfer 12 (1973) 25-40.

[16] R. Siegel, J.R. Howell, Thermal Radiation Heat Transfer, 3rd ed., Hemisphere, Washington, DC, 1992 (chap. 13).

[17] P.J. Foster, C.R. Howarth, Optical constants of carbons and coals in the infrared, Carbon 6 (1968) 719-729.

[18] M.P. Menguc, R. Viskanta, Radiative transfer in threedimensional rectangular enclosures containing inhomo- 
geneous, anisotropically scattering media, J. Quant. Spectros. Radiat. Transfer 33 (1985) 533-549.

[19] S. Maruyama, Radiation heat transfer in anisotropic scattering media with specular boundary subjected to collimated irradiation, Int. J. Heat Mass Transfer 41 (1998) 2847-2856.

[20] Z. Guo, S. Maruyama, Scaling anisotropic scattering in radiative transfer in three-dimensional nonhomogeneous media, Int. Commun. Heat Mass Transfer 26 (1999) 997-1007.

[21] J.C. Chai, H.S. Lee, S.V. Patankar, Ray effect and false scattering in the discrete ordinates method, Numerical Heat Transfer, Part B 24 (1993) 373-389.
[22] T. Omori, S. Murakami, S. Katoh, C.K. Choi, H. Kobayashi, Coupled simulation of convective and radiative heat transfer in enclosed space: accuracy of shape factors obtained by Monte Carlo method, in: Proc. Air-Con. Sanit. Eng. Japan, 1992, pp. 653-656.

[23] H. Aoki, S. Tanno, T. Miura, S. Ohnishi, Three-dimensional spray combustion in a practical boiler, JSME Int. J., Ser. II 35 (1992) 428-434.

[24] K.C. Smyth, J.H. Miller, R.C. Dorfman, W.G. Mallard, A.R. Santoro, Soot inception in a methane/air diffusion flame as characterized by detailed species profiles, Combust. and Flame 62 (1985) 157-181. 